\title{
Miniature Filters, Diplexers and Antennas of L-Band Used in Receivers of Satellite Radionavigation System
}

\author{
Babus hkina O., Golovkov A., Kers his S., Kuzmenko V." \\ Saint Petersburg Electrotechnical University "LETI", Russia
}

\begin{abstract}
New miniature filters, diplexers, based on low and high pass filter, and antennas for satellite radio navigation system based on low and high pass filter are presented. These devises are manufactured on ceramic substrates with different thickness and $\varepsilon=40,80,120$ by using thick-film technology where topology is formed with silver conductors by laser.In the article simulated and experimental filters, diplexers and antennas main characteristics are pres ented as well as a topology
\end{abstract}

Keywords Filters, Antennas, Diple xers, GLONASS, GPS

\section{Introduction}

In many practical cases while satellite radio navigation system consumer equipments are implemented (GLONASS and GPS) frequency selective circuits and antennas realized on ceramic substrates are often used[5-13]. These devices have very high vibro-, heat and radiation resistance and work under the influence of very high electromagnetic pulse power. Because of new types of very-Q radio ceramic with high inductive capacity $(\varepsilon \sim 80 \ldots 120)$ that have been recently explored these devises might be imple mented very s mall size compared with discrete units applied at radio receiving equipment and oriented for SMD technology manufacturer. There have been worked out single, double bandpass filters, antennas and diplexers for L1, L2 bands that are going to be launched in serial production[1-3]. These devises are manufactured on ceramic substrates with different thicknesses and $\varepsilon=40,80$ by using thick-film technology where topology is formed with silver conductors by laser. Frequency selective units were made for installation by using SMD technology and had output ports located on the under-side of the substrate.

\section{Filters}

Bandpass filters and diplexers were designed with help of AWR "Microwave Office" in two steps. In the first the primary structure optimization was carried on schematic level. In the second - on layout level by using the EMSight solver for structure calculation.

* Corresponding author:

vk@transtronics.ru (Kuzmenko V.)

Published online at http://journal.sapub.org/eee

Copyright (C) 2012 Scientific \& Academic Publishing. All Rights Reserved
When value of conductive capacity $\varepsilon$ reaches 40,80 because of existing high frequency modes insight substrate it might be seen differences in simulated results that is why in the second step there was manual optimization made. In addition at topology creation by laser at the depth 3-5 u m a high substrate layout is deleted. In that case repetitive simulation was made with tools of Ansoft HFSS which allowed to examine the effect carefully. Simulated and measured characteristic coincidence was satisfactory after the steps mentioned earlier and dependent on $\varepsilon$ variation and specific wire conductivity.

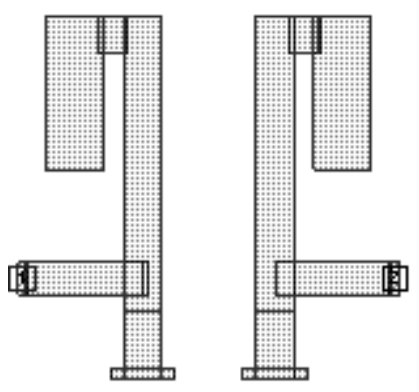

(a)

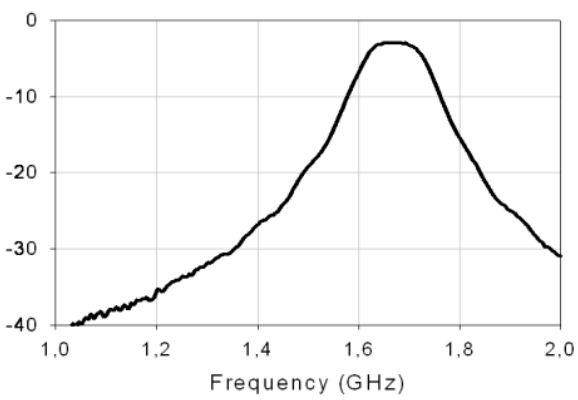

(b)

Figure 1. The $\lambda / 4$ filter's topology (a) and measured $\left|S_{21}\right|$ for this filter (b)

Bandpass filters have been designed on the basic of $\lambda / 4$ and $\lambda / 2$ step-impedance resonators to work at L1 and L2 bands of satellite radio navigation system. 
Quarter-wave-length step-impedance resonators have the same structure and topology but different physical sizes. The topology of this filter designed for a combined receiver working in both GLONASS and GPS diapasons is shown in figure 1(a).

The size of the filter designed for L1 band is $4.5 \times 4 \times 0.5 \mathrm{~mm}^{3}$, for $\mathrm{L} 2$ band $-4.5 \times 5 \times 0.5 \mathrm{~mm}^{3}$. Measured $\left|\mathrm{S}_{21}\right|$ of filter for L1 band is represented in figure 1(b).

The bandpass filters realized on half-wave-length step-impedance resonators have smaller loss es in bandwidth but a little bit bigger sizes than filters described above. Topology of these filters is shown in figure 2.

Just as the previous case filters have the same structure but different physical sizes $\left(7 \times 8 \times 0.5 \mathrm{~mm}^{3}-\mathrm{L} 1,9 \times 8 \times 0.5 \mathrm{~mm}^{3}-\right.$ L2). The measured frequency responses of both filters are shown in figure $3 a, b$.

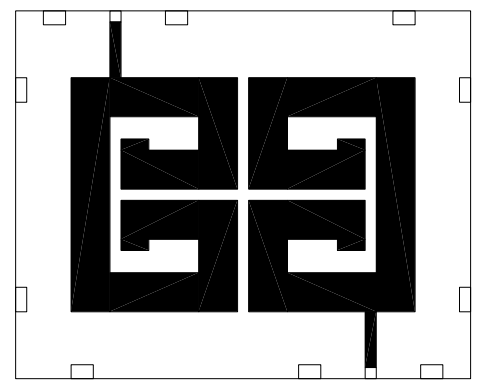

Figure 2. The $\lambda 2$ filter's topology

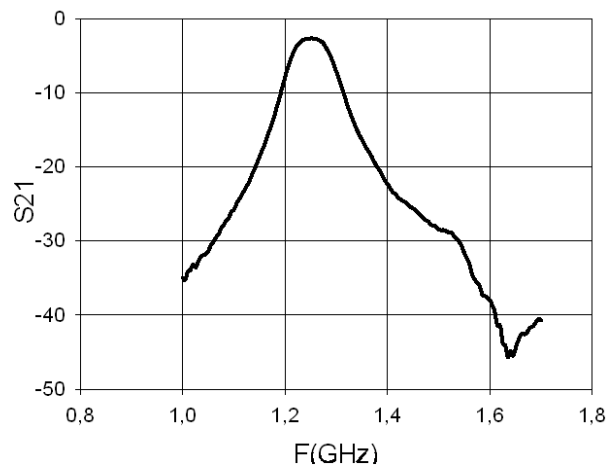

(a)

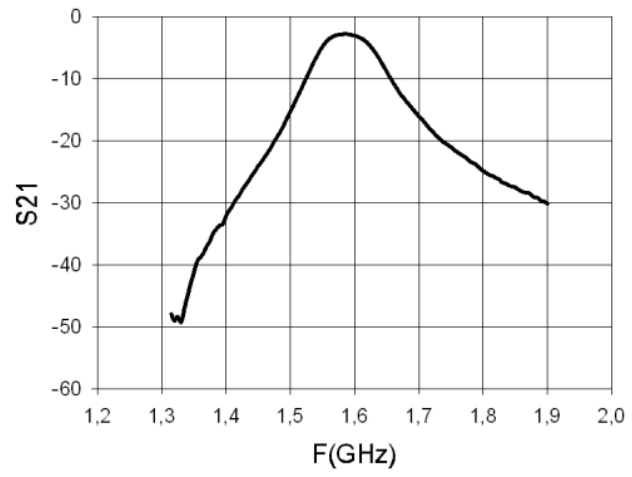

(b)

Figure 3. The experimental $\lambda / 2$ filter's main charact eristics

In many cases double band devices of satellite radio navigation system take signals out of one antenna. The main distortion component located in the desired signal spectrum near L1 and L2 bands are signals of mobile system. In real situation these signals exceed navigation signal at $70 \mathrm{db}$. That means there must be a filter having got zeros of frequency response at mobile frequencies between the antenna and receiver input. Implementation one of the filters built on $\mu$-shaped double-frequency resonators is shown in figure 4(a).

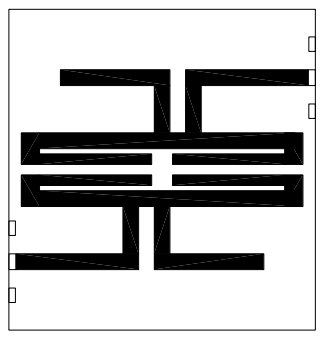

(a)

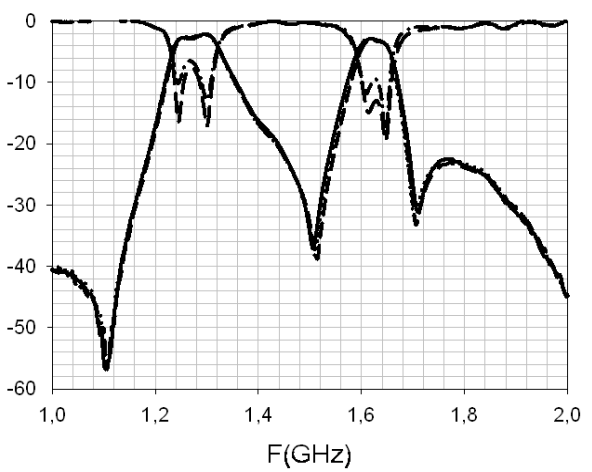

(b)

Figure 4. The two-band filter's topology (a) and filters main characteristics

The simplest case of that when the double-frequency resonator consists of two $\lambda / 2$ resonators united symmetrically with common point of intersection.

This double-frequency filter has size $11 \times 10 \times 0.5 \mathrm{~mm}^{3}$. Measured frequency response characteristics of several pattern of the filter are shown in figure 4(b). On the graphs discussed filters have additional zeros at the frequencies which are often used in other telecommunication systems.

Described filters have good frequency response repetition, easy and cheap in manufacture and installation on the board of navigation receiver.

\section{Diplexers}

A structure of low pass filter (LPFpf) which has got very broad stopband was suggested in the article[4]. In the article [1] further development of the structure for UHF based on UHF distributed phase circuit loaded on open transmission line was analysed. In the article[2] the structure based on UHF distributed phase circuit was investigated in case of changing open transmission line for short transmission line as a load. The main important feature of all structures described above was absence of additional frequency response zeros beyond filter bandpass. Analys is 
showed that changing physical length and impedance of transmission line leads to locate the frequency response zeros right at the central frequency of band GLONASS and GPS and therefore to get maximum dip lexer's selectivity in the specific frequency range. The first variant of diplexer to separate different navigation signals (GLONASS, GPS) coming from one antenna of navigation receiver used combination LPF and HPF examined in the articles [1, 2]. However that diplexer had low impedance because of broad conductors on the substrate with $\varepsilon=80$. In order to match it to standard impedance there was an additional transforming chain in the diplexer scheme. A topology of that diplexer is shown in figure 5 . Printed circu it board size is $15 \times 10 \times 0.4$ $\mathrm{mm}^{3}$

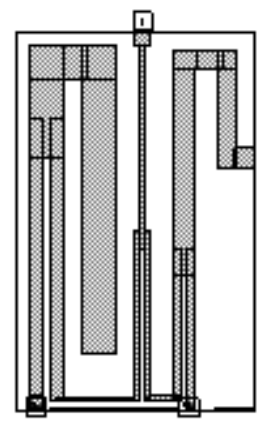

Figure 5. The first variant of diplexer topology

Simulated points and measured frequency response characteristics $\left|S_{21}\right|$ and $\left|S_{31}\right|$ are shown in figure 6a,b.

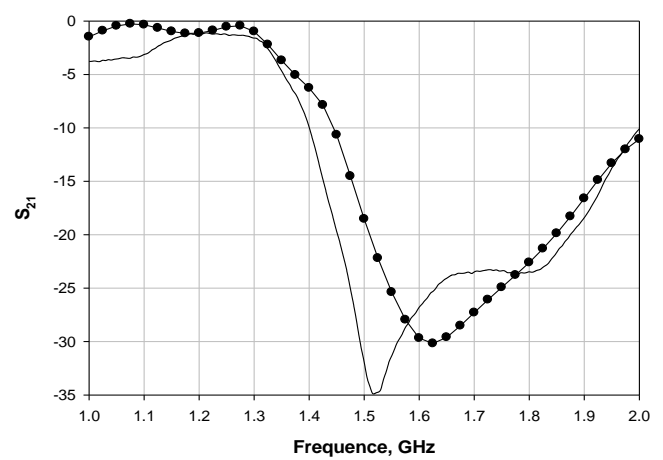

(a)

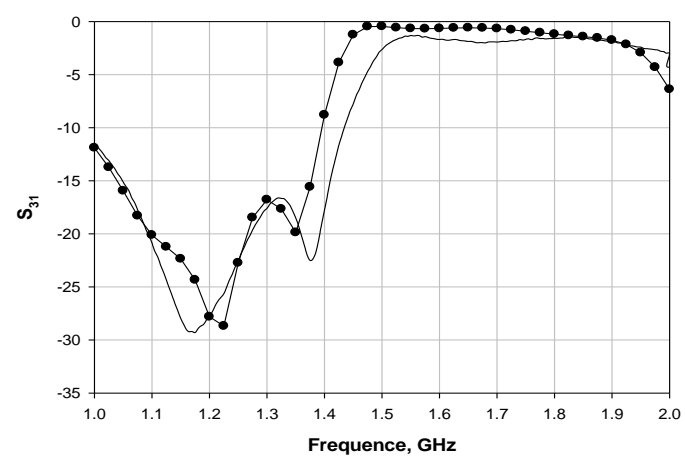

(b)

Figure 6. Diplexer's main characteristics
The second variant of diple xer was built on quarter wave length step-impedance resonators. It had very high suppression of distortion of mobile system and unfortunately a little bit losses in desired bandpasses of L1 and L2 (GPS, GLONASS). A topology of that diplexer and measured data of its characteristics are shown in figure 7 . Printed circu it board size is $13 \times 6.5 \times 0.5 \mathrm{~mm}^{3}$.

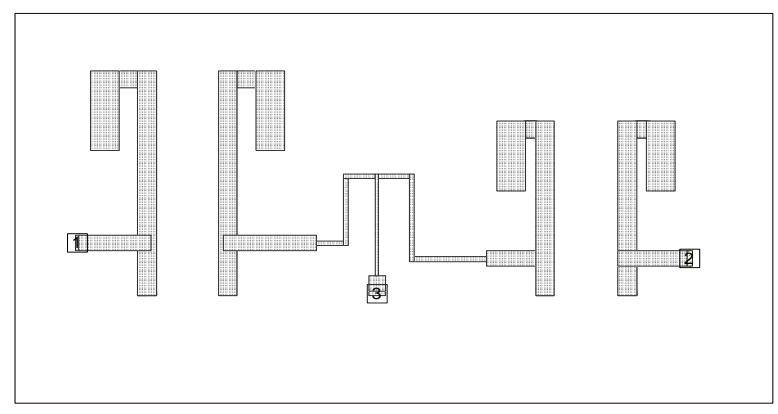

(a)

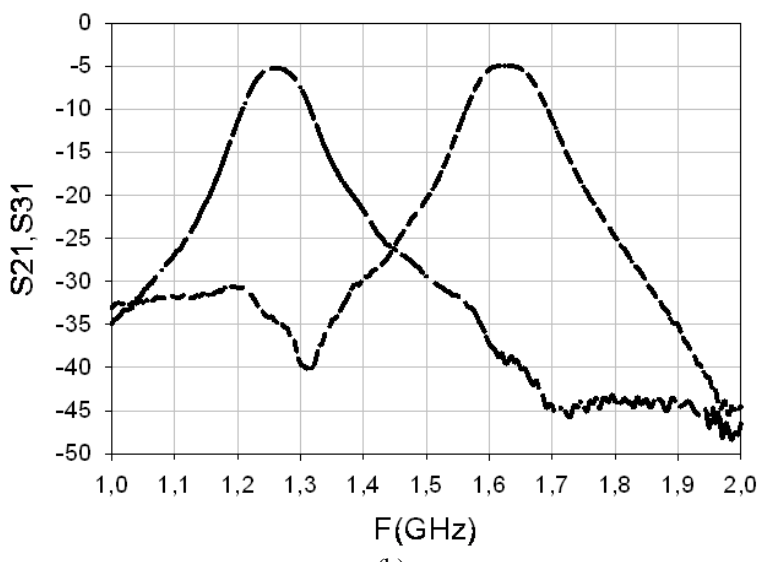

(b)

Figure 7. The second variant of diplexer main characteristics

Good repetition of filter characteristics also allowed realizing the third variant of diplexer for separating navigation signals. It differed from others because has much more improved features to suppress distortion of mobile system. Topology of that diplexer is shown in figure 8 . Printed circuit board size is $17 \times 8 \times 0.5 \mathrm{~mm}^{3}$.

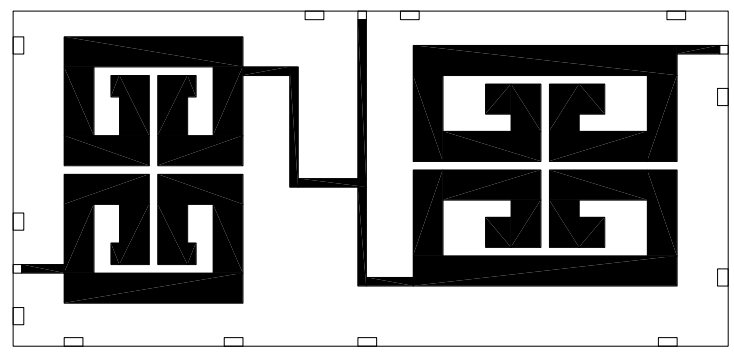

Figure 8. The third variant of diplexer topology

Calculated frequency response characteristics of the diplexer build on half wave length step-impedance resonators and measured data is shown in figure 9. 


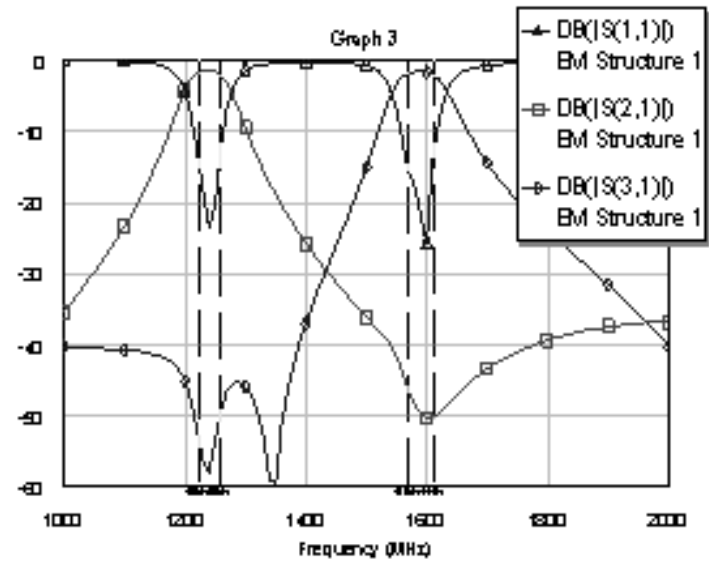

(a)

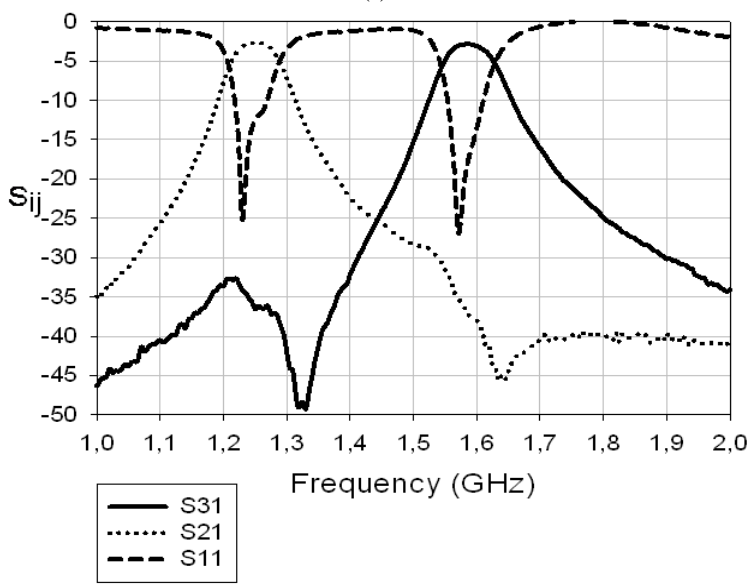

(b)

Figure 9. The third variant of diplexer main characteristics

\section{Antennas}

For satellite radio navigation system consumer equip ment (GLONASS and GPS) several variants of ceramic antennas which has small sizes and are able to receive signal with right-hand circular polarization in L1 and L2 bands has been also developed. Antenna design was made in Ansoft HFSS. Simulation examined bands were shifted from measured ones at 3-5 MHz and the final antenna conductor sizes were selected manually on base of experienced data.

A single-frequency antenna looks like rectangular patch antenna that has chamfers on both opposite site of conductor. These chamfers ensure link between two modes excited in the patch antenna and allows to receive signal with circular polarization. A topology of the antenna is shown in figure 10(a). It was built on substrate with $\varepsilon=40$ and thickness equals $4 \mathrm{~mm}$. Antenna size for L1 band is $18 \times 18 \times 5 \mathrm{~mm}^{3}$, for

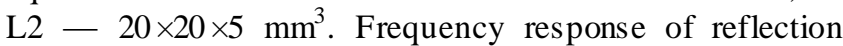
coefficient of the antenna is shown in figure $10(\mathrm{~b})$.

The peak directivity of this antenna is $2 \mathrm{~dB}$. Measured antenna efficiency was about $75 \%$. The antenna of L2 band has the close characteristic to the antenna of L1 band.

Similar to each other from the point of view of their structure the single-frequency antennas implemented on substrate with $\varepsilon=80$ have sizes $13.2 \times 13.2 \times 5 \mathrm{~mm}^{3}$ for $\mathrm{L} 1$ and
$17 \times 17 \times 5 \mathrm{~mm}^{3}$ for $\mathrm{L} 2$ band. Measured data showed efficiency of these antennas is about $65 \%$.
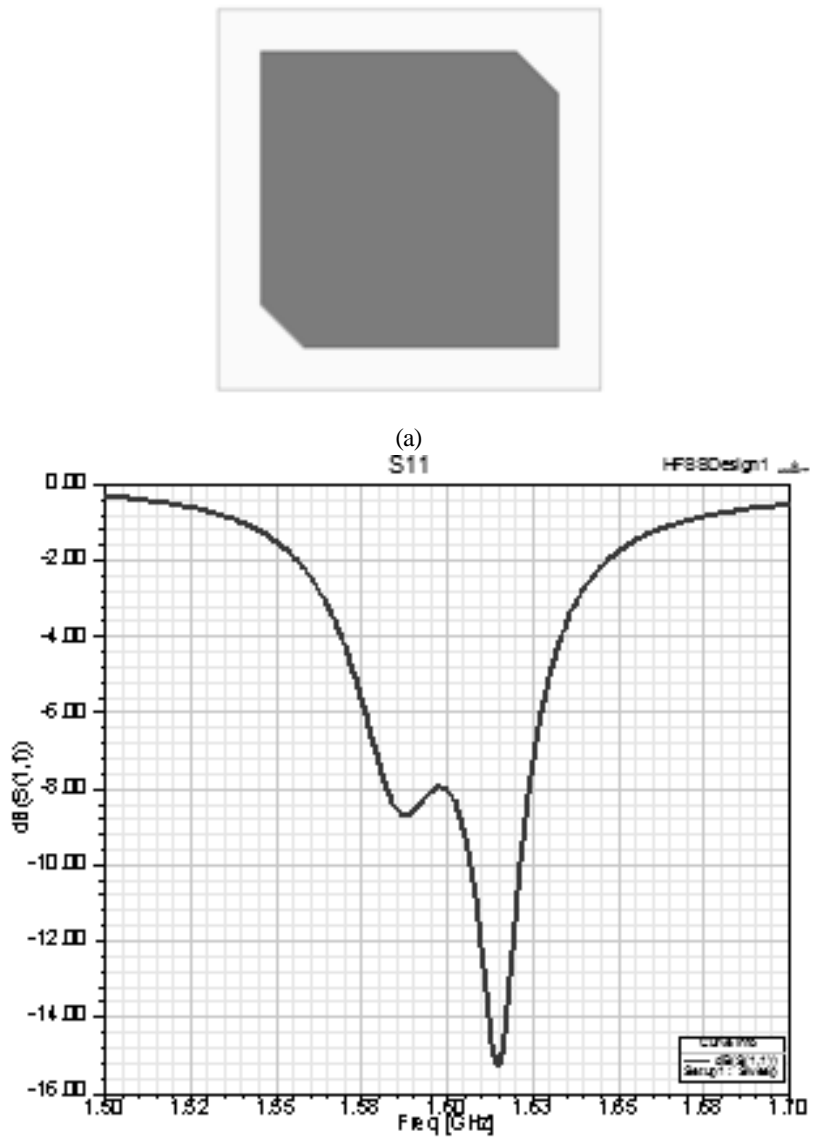

(b)

Figure 10. A topology of the antenna and its frequency response of reflection coefficient

In order to receive navigation signal of different bands L1 and L2 at the same time multilayer a double-frequency antenna was implemented, structure of which is shown in figure 11. The top layer was built to catch the signal of L2 band and the bottom was built to catch the signal of L1 band. Like antenna construction discussed earlier the chamfers at the end of the conductor give opportunity to get signal with right-hand circular polarization without using a splitter.

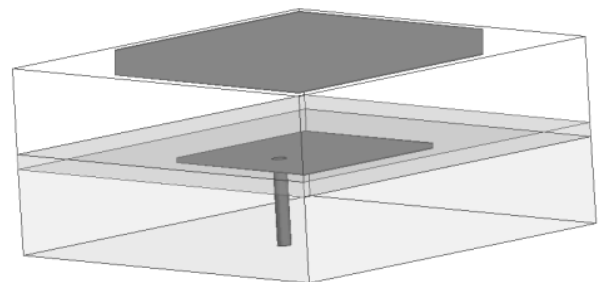

Figure 11. The multilayer antenna to receive navigation signal of dual L1 and $\mathrm{L} 2$ bands

Conductors were made on substrates with $\varepsilon=40$ and thickness $6 \mathrm{~mm}$. Between two layers one more layer was located with $\varepsilon=2.6$ and thickness $1 \mathrm{~mm}$. Size of the antenna was $28 \times 28 \times 13 \mathrm{~mm}^{3}$. Frequency response reflection coefficient of that antenna is shown in figure 12 . 


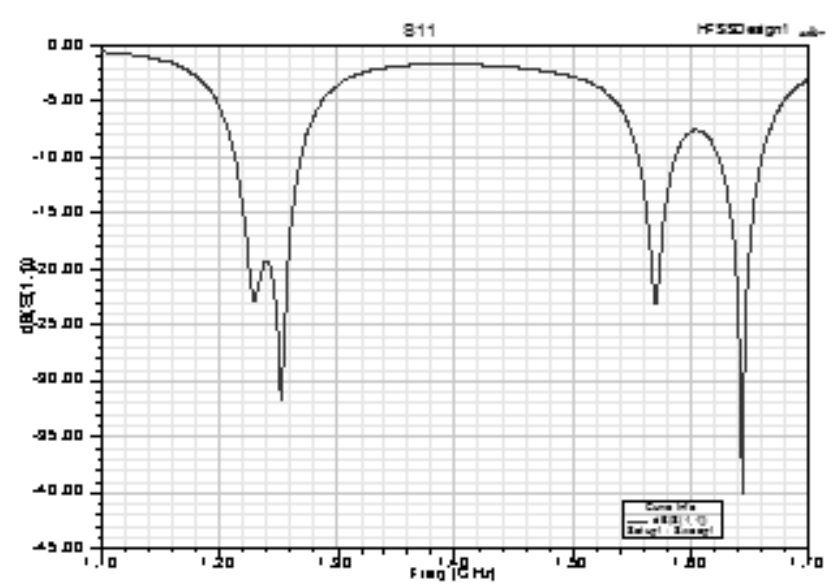

Figure 12. Frequency response reflection coefficient of the multilayer antenna

Directivity of that antenna in E plane $(\theta=0)$ for right-hand circular polarization at the frequencies $1.23 \mathrm{GHz}$ and 1.6 $\mathrm{GHz}$ are shown in figure $13(\mathrm{a})$ and 13 (b) respectively.
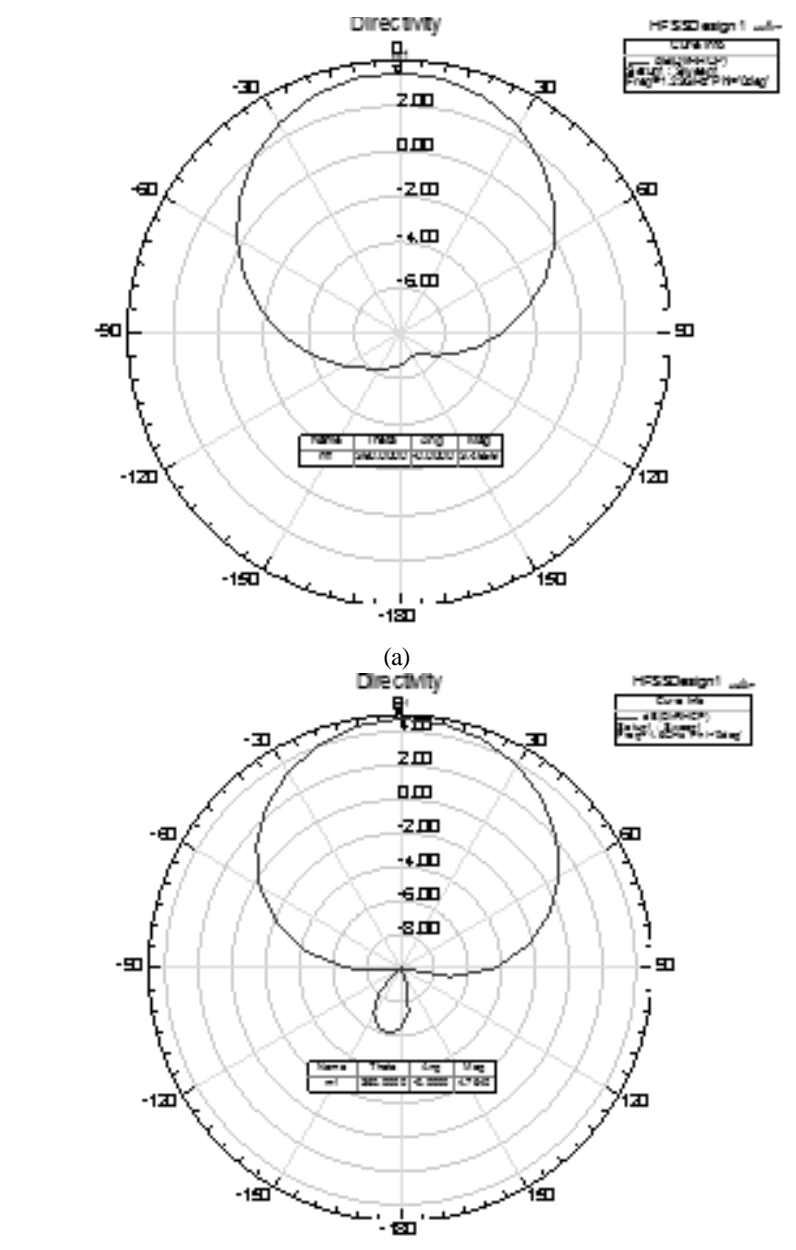

(b)

Figure 13. Antenna directivity at the frequencies $1.23 \mathrm{GHz}(\mathrm{a})$ and $1.6 \mathrm{GHz}(\mathrm{b})$

As the result new antennas small in size and easy in manufacture were made and examined. These antennas can catch navigation signals of circular polarization of both L1 and L2 bands.

\section{Conclusions}

In the article were suggested simulation data of filters small sizes, diplexers and antennas for satellite radio navigation system GPS and GLONASS implemented on the substrate with high value of inductive capacity $\varepsilon$ and expected for installation with help of SMD technology. Results got during execution and analysing says that the device sizes were too smaller than[14], but had losses higher than[14]; they had losses the same as[15] but had smaller sizes.

\section{REFERENCES}

[1] Babushkina O, Golovkov A. UHF passband filters with the possibility to suppress spurious bandwidths/ Radioelectronics questions, ser. Radiolocation engineering, pub.4, 2008, p.51-59.

[2] Babushkina O, Kershis S. UHF Highpass filters with ultrabroad stopband./ SPbGETU "LETI" news, pub.2, 2011, p.8-12.

[3] Very small filters and diplexers for satellite radio navigation system GLONASS and GPS /Babushkina O, Golovkov A., Kershis S.,Kuzmenko V.,Pivovarov U.//Proceedings of 21 Crimea's International conference "UHF technics and telecommunication technologies", september 12-16, 2011, Sevastopol, Ukraine, T.2, p.610-612.

[4] Rui Li, Dong Kim, Chang Mook Choi. Compact Structure With Three Attention Poles for Improving Stopband Characteristics - IEEE Microwave and Wireless Components Letters,Vol. 16, NO. 12, December, 2006, p.663.

[5] An Ultra-Compact Hairpin Band Pass Filter with Additional Zero Points /Kaixue Ma, Kiat Seng Yeo, Jianguo Ma and etc. // IEEE Microwave and Wireless Components Letters,Vol. 17, NO. 4, April, 2007, p.262.

[6] LinS.C., Wang C.Y., Chen C.I.. Novel Patch-Via-Spiral Resonators for Development of Miniaturised Bandpass Filters with Transmission Zeros // IEEE Transaction on Microwave Theory and Techniques, Vol. 55, NO.1, January, 2007, p.155.

[7] Yao B., Zhou Y., Cao Q., Chtn Y. Compact UWB Bandpass Filter with Improved Upper-Stopband Performance // IEEE Microwave and Wireless Components Letters, Vol.19, N1, p.27-29, Jan., 2009.

[8] Lok U.-H., Chiou Y.-C., Kuo J.-T. Quadruple-Mode Coupled-Ring Resonator Bandpass Filter with Quasi-Elliptic Function Passband // IEEE Microwave and Wireless Components Letters, Vol.18, N3, p.179-182, Mar., 2008.

[9] Zhang X.-C., Yu Z.-Y., Xu J. Design of Microstrip Dual-Mode Filters Based on Source-Load Coupling // IEEE Microwave and Wireless Components Letters, Vol.18, N9, p.677-680, Oct, 2008.

[10] Zhu J., Feng Z.Microstrip interdigital Hairpin Resonator with an Optimal Phy sical Length // IEEE Microwave and Wireless Components Letters, Vol.16, N12, p.672-675, Dec., 2006. 
[11] Tang C.-W., Liu W.-T. Design of Wide Stopband Microstrip Bandpass Filter with Interdigital Resonators // IEEE Microwave and Wireless Components Letters, Vol.18, N12, p.767-770, Dec, 2008.

[12] Tang C.-W., Ysu Y.-K. Design of Wide Stopband Microstrip Bandpass Filter with Asymmetrical Resonators // IEEE Microwave and Wireless Components Letters, Vol.18, N2, p.91-94, Feb., 2008.
[13] Chun Y.-H., Hong J.-S. Electronically Reconfigurable Dual-Mode Microstrip Open-Loop Resonator Filter // IEEE Microwave and Wireless Components Letters, Vol.18, N7, p.449-452, Jul., 2008.

[14] K\&L GPS Miniature Ceramic Diplexer. Available: www.klmicrowave.com

[15] .DLI GPS Notch Filter and GPS Ceramic Diplexer. Available: www.dilabs.com 